\title{
Relative performance of indoor vector control interventions in the Ifakara and the West African experimental huts
}

\author{
Welbeck A. Oumbouke ${ }^{1 *}$, Augustin Fongnikin ${ }^{1}$, Koffi B. Soukou', Sarah J. Moore ${ }^{2,3,4}$ and Raphael N'Guessan ${ }^{1,5}$
}

\begin{abstract}
Background: West African and Ifakara experimental huts are used to evaluate indoor mosquito control interventions, including spatial repellents and insecticides. The two hut types differ in size and design, so a side-by-side comparison was performed to investigate the performance of indoor interventions in the two hut designs using standard entomological outcomes: relative indoor mosquito density (deterrence), exophily (induced exit), blood-feeding and mortality of mosquitoes.

Methods: Metofluthrin mosquito coils (0.00625\% and 0.0097\%) and Olyset ${ }^{\oplus}$ Net vs control nets (untreated, deliberately holed net) were evaluated against pyrethroid-resistant Culex quinquefasciatus in Benin. Four experimental huts were used: two West African hut designs and two Ifakara hut designs. Treatments were rotated among the huts every four nights until each treatment was tested in each hut 52 times. Volunteers rotated between huts nightly.

Results: The Ifakara huts caught a median of 37 Culex quinquefasciatus/ night, while the West African huts captured a median of 8/ night (rate ratio 3.37, 95\% Cl: 2.30-4.94, $P<0.0001$ ) and this difference in mosquito entry was similar for Olyset $^{\oplus} \mathrm{Net}$ and more pronounced for spatial repellents. Exophily was greater in the Ifakara huts with $>4$-fold higher mosquito exit relative to the West African huts (odds ratio 4.18, 95\% Cl: 3.18-5.51, $P<0.0001$ ), regardless of treatment. While blood-feeding rates were significantly higher in the West African huts, mortality appeared significantly lower for all treatments.

Conclusions: The Ifakara hut captured more $C X$. quinquefasciatus that could more easily exit into windows and eave traps after failing to blood-feed, compared to the West African hut. The higher mortality rates recorded in the Ifakara huts could be attributable to the greater proportions of Culex mosquitoes exiting and probably dying from starvation, relative to the situation in the West African huts.
\end{abstract}

Keywords: Experimental hut, Mosquito, Long-lasting insecticidal net, Mosquito coil, West African hut, Ifakara hut, Benin

\section{Background}

During the Global Malaria Eradication Campaign (1955-1969) the need to evaluate the efficacy of residual insecticides such as DDT and Dieldrin for malaria campaigns motivated the development of experimental huts. The so-called West African experimental hut also referred to as "verandah trap hut" first built in Burkina Faso is a modified version of the huts designed by Smith \& Hudson [1]. The design is based upon that of modern brick-walled houses with corrugated iron roofs. This

\footnotetext{
* Correspondence: achiloswek@yahoo.fr

${ }^{1}$ Centre de Recherche Entomologique de Cotonou, Cotonou, Benin

Full list of author information is available at the end of the article
}

type of experimental hut is included in WHOPES (World Health Organization Pesticide Evaluation Scheme) guidelines mainly because it is based on the design of local houses in Burkina Faso, simple and cheap to construct but also because it requires simple entomological collection methods [2]. Mosquitoes enter these huts through small window slits located on three sides and are trapped within the huts or in a largely walled verandah projected from the fourth side of the huts. There is a curtain between the room and the verandah that is usually raised during the night and lowered in the morning to prevent mosquitoes from flying back into the room. The hut allowed the assessment and 
comparison of several products against free flying malaria vectors and the West African design is now found in several African countries, including Burkina Faso, Benin, Cameroon and even Asia (Vietnam).

The Ifakara hut design [3] is a modified version of the "Portable hut" designed in Belize, Central America [4] that has similar dimensions to Tanzanian homes and includes features of East African hut designs including baffles to prevent mosquito egress [5], window traps [6] but with eave traps rather than verandah traps to capture mosquitoes that exit through eave gaps [7]. Both the West African and Ifakara huts are used to evaluate in WHO phase II trials the efficacy of mosquito control interventions, including insecticide [8-10] and repellentbased products $[11,12]$. Though these huts have been in use for many years, only one study has determined the difference in LN efficacy that occur due to hut structure. This study was performed in Tanzania and compared the performance of Olyset $^{\circ}$ Net LN in three huts types, East African huts, Ifakara huts and West African experimental huts [13]. The East African and Ifakara huts were shown to capture substantially more mosquitoes, compared to the West African design. However, due to the high mortality in control huts (>40\%) recorded in the Tanzanian trial, it was not possible to compare net efficacy between huts types. Therefore, the question of whether the efficacy of a candidate indoor intervention changes with experimental hut design remains unaddressed.

Because they differ in design, each hut style may have benefits or weaknesses depending on the characteristics of the intervention under evaluation. This study aimed to compare the efficacy of a standard insecticidal net (Olyset ${ }^{\circ}$ Net) and a mosquito repellent coil (metofluthrin), both evaluated in each type of huts side-by-side in Benin.

\section{Methods}

\section{Study site}

The study was conducted at Donoukin, a suburban village on the outskirts of Porto Novo, the administrative capital of Benin. The site supports the breeding of Anopheles gambiae $\mathrm{M}$ form, mostly pyrethroid-resistant with a high frequency of $k d r(>90 \%)$ and increased activity of cytochrome P450s [14]. The nuisance mosquito Culex quinquefasciatus is present in abundance year round and shows resistance to pyrethroids, carbamates and organophosphates [15].

\section{Experimental huts}

Two West African and two Ifakara huts were constructed approximately $50 \mathrm{~m}$ apart from one another in a row between mosquito breeding sites and human habitations to maximize interception of host-seeking mosquitoes. Huts of the same design were constructed in a row, and the West African huts were located behind the Ifakara experimental huts.

The West African experimental huts are $2.5 \mathrm{~m}$ long $\times 1.75 \mathrm{~m}$ wide $\times 2 \mathrm{~m}$ high. The walls are made of concrete bricks and the roof of corrugated iron. A plastic cover is stretched under the roofing as a ceiling to facilitate the catching of mosquitoes. Entry of mosquitoes occurs through four slits, $1 \mathrm{~cm}$ wide, located on three sides of the hut. These slits were designed to prevent mosquitoes from escaping once they entered the hut. Mosquitoes can egress into a large verandah trap projecting from the fourth side. Each hut stands on a concrete base surrounded by a water-filled moat to exclude scavengers such as ants and spiders.

The Ifakara experimental huts design are $6.5 \mathrm{~m}$ long $\times$ $3.5 \mathrm{~m}$ wide $\times 2 \mathrm{~m}$ high. The walls are made of canvas lined with reed matting with a corrugated iron roof and a $10 \mathrm{~cm}$ eave gap on all sides through which mosquitoes enter and are prevented from leaving by netting baffles. Mosquito entry points are interspersed with eight eave interception traps fitted to huts on each side to catch eave-exiting mosquitoes in addition to four window exit traps: two on the front and two on the back of the huts. The huts are suspended above ground using concrete pillars surrounded by a water-filled moat to keep predators away from mosquitoes.

\section{Interventions}

Control represented untreated white 100-denier polyester multifilament net (Siam-Dutch Mosquito Netting Co., Bangkok, Thailand) deliberately holed with six holes $(4 \times 4 \mathrm{~cm})$ cut along the sides to simulate a torn net.

Olyset $^{\circ}$ Net is a long-lasting insecticidal net (LLIN), made of knitted polyethylene thread with permethrin at $20 \mathrm{~g} / \mathrm{kg}(2 \% \mathrm{w} / \mathrm{w})$ incorporated in it (Sumitomo Co, Tokyo, Japan). Both the control net and the Olyset net were deliberately holed with 6 holes $(4 \times 4 \mathrm{~cm})$ cut along the sides to simulate wear and tear as per WHO guidelines [16].

Two doses of metofluthrin mosquito coils were evaluated: $0.00625 \%$ and $0.0097 \%$ (SC Johnson and Sons, USA). These were tested in conjunction with untreated, deliberately holed nets. One coil was used per hut per night for each of the two doses and burned for about $8 \mathrm{~h}$.

\section{Study procedure}

The study was a partially randomised Latin Square design where the four treatments were rotated weekly between the four huts (two West African huts and the two Ifakara designs) and volunteer sleepers rotated on a nightly basis to compensate for any variation in individual attractiveness. The study was conducted over 20 weeks with eight initial weeks between November $16^{\text {th }}, 2011$ and January $14^{\text {th }}, 2012$ followed by a further 
12 weeks between June $13^{\text {th }}, 2012$ to September $2^{\text {nd }}$, 2012. Each treatment spent 52 nights in each hut. Each intervention was tested in each hut an equal number of times, i.e. 52 nights.

Four volunteer sleepers from nearby villages were recruited on written informed consent to sleep in the huts from 20:00 to 05:00 h each night. The area is highly endemic for malaria; sleepers were screened and treated for malaria, although no one fell ill during the study. The coil was lit at 20:00 h and was kept at a distance of $1 \mathrm{~m}$ away from the sleeper. In the morning, each volunteer collected mosquitoes from the walls, floors, under bed nets, ceilings, verandahs, and traps of the hut using torches and aspirators. Mosquitoes were transported to the laboratory for identification to species and scored as alive or dead, blood-fed or unfed. Surviving mosquitoes were held in plastic cups supplied with $10 \%$ glucose solution. The temperature was maintained at $27 \pm 2{ }^{\circ} \mathrm{C}$ and relative humidity at $75 \pm 10 \%$. Mortality was scored $24 \mathrm{~h}$ post holding.

\section{Outcomes}

The impact of each treatment was assessed according to the following parameters: (i) deterrence: percentage reduction in the number of mosquitoes caught in treated hut relative to the number caught in the control hut; (ii) induced exophily: percentage of the mosquitoes collected from exit traps of treated hut relative to percentage caught in exit traps of control hut; (iii) blood-feeding rate: percentage of the mosquitoes collected that were blood-fed in experimental huts; (iv) induced mortality: percentage of dead mosquitoes in treated hut relative to percentage dead in control hut; and (v) personal protection: the proportional reduction in the number of blood-fed mosquitoes relative to blood-fed mosquitoes in the control group.

\section{Data analysis}

Data were collected on paper forms and entered into an Excel data base. Data were analyzed using the $\mathrm{R}$ statistical software version 2.15 .0 [17] with a significance level of 0.05 for rejecting the null hypothesis. All generalized linear mixed models (GLMMs) were conducted using the lme4 package [18]. Count data were modelled using a generalised linear model with a poisson distribution and logit link with treatment position, sleeper and day treated as random effects; treatment and hut type were fixed effects and fitted with an interaction. Proportional data (mortality, exophily and blood-feeding) were analysed using a generalised linear model with a binomial distribution and a logit link with position, and day treated as random effects; treatment, hut type and sleeper were fixed effects with treatment and hut type fitted with an interaction. Several GLMMs were run for each outcome and the final model selected was that with the lowest Akaike's information criterion (AIC). Also, residuals were plotted using a histogram, quantile quintile plots and comparison with fitted values to ensure appropriateness of model selection.

\section{Results}

A total of $7655 C x$. quinquefasciatus females were collected in the huts, of which 6263 were caught in the Ifakara huts and 1392 in the West African hut designs. Only 381 and 35 Anopheles were collected in the Ifakara and West African huts, respectively. Given the low numbers collected meaningful comparisons were difficult and so only $C x$. quinquefasciatus data were analyzed and reported.

\section{Mosquito density}

Overall, the Ifakara huts captured considerably more Cx. quiquefasciatus than the West African huts, whether or not they contained a product (Tables 1 and 2). The median in the Ifakara hut with control untreated net was 37 , and the inter quartile range

Table 1 Deterrence of Cx. quinquefasciatus induced by Olyset ${ }^{\oplus}$ Net, metofluthrin coils at $0.00625 \%$ and $0.0097 \%$ and untreated control net in Ifakara and West African experimental huts

\begin{tabular}{|c|c|c|c|c|c|c|c|}
\hline & No. caught & Median IQR caught & IRR & $95 \% \mathrm{Cl}$ & $P$-value & $Z$ & Deterrence $\%$ \\
\hline \multicolumn{8}{|l|}{ Ifakara huts } \\
\hline Control net & 2067 & $37(27-51)$ & 1 & & & & - \\
\hline Metofluthrin $0.00625 \%$ & 1489 & $23(15-36)$ & 0.61 & $0.56-0.66$ & $<0.0001$ & -12.38 & 28 \\
\hline Metofluthrin $0.0097 \%$ & 1054 & $21(11-29)$ & 0.65 & $0.59-0.71$ & $<0.0001$ & -9.55 & 49 \\
\hline Olyset $^{\oplus}$ Net & 1658 & $29.5(18.5-37.5)$ & 0.82 & $0.75-0.90$ & $<0.0001$ & -4.02 & 20 \\
\hline \multicolumn{8}{|l|}{ West African huts } \\
\hline Control net & 587 & $8(4-14.5)$ & 1 & & & & - \\
\hline Metofluthrin $0.00625 \%$ & 122 & $1(0-4)$ & 0.25 & $0.20-0.30$ & $<0.0001$ & -13.89 & 79 \\
\hline Metofluthrin $0.0097 \%$ & 130 & $1(0-4)$ & 0.24 & $0.14-0.21$ & $<0.0001$ & -17.75 & 78 \\
\hline Olyset $^{\oplus}$ Net & 553 & $7.5(3-17.5)$ & 0.84 & $0.73-0.96$ & 0.01 & -2.48 & 6 \\
\hline
\end{tabular}


Table 2 Measurements of deterrence compared between Ifakara and West African experimental huts for control net, Olyset ${ }^{\circledR}$ Net and metofluthrin coils at $0.00625 \%$ and $0.0097 \%$

\begin{tabular}{|c|c|c|c|c|c|c|c|}
\hline & No. caught & Median IQR caught & RR & $95 \% \mathrm{Cl}$ & $P$-value & $Z$ & Deterrence $\%$ \\
\hline \multicolumn{8}{|l|}{ Control net } \\
\hline West African hut & 587 & $8(4-14.5)$ & 1 & & & & - \\
\hline Ifakara hut & 2067 & $37(27-51)$ & 3.37 & $2.30-4.94$ & $<0.0001$ & 6.23 & - \\
\hline \multicolumn{8}{|l|}{ Olyset $^{\oplus}$ Net } \\
\hline West African hut & 553 & $7.5(3-17.5)$ & 1 & & & & 6 \\
\hline Ifakara hut & 1658 & $29.5(18.5-37.5)$ & 3.29 & $2.24-4.84$ & $<0.0001$ & 6.08 & 20 \\
\hline \multicolumn{8}{|c|}{ Metofluthrin $0.00625 \%$} \\
\hline West African hut & 122 & $1(0-4)$ & 1 & & & & 78 \\
\hline Ifakara hut & 1489 & $23(15-36)$ & 8.58 & $5.68-12.96$ & $<0.0001$ & 10.21 & 28 \\
\hline \multicolumn{8}{|c|}{ Metofluthrin $0.0097 \%$} \\
\hline West African hut & 130 & $1(0-4)$ & 1 & & & & 79 \\
\hline Ifakara hut & 1054 & $21(11-29)$ & 12.69 & $8.40-19.15$ & $<0.0001$ & 12.09 & 49 \\
\hline
\end{tabular}

(IQR) 27-51 Culex mosquitoes per hut per night compared to 8 (IQR 4.0-14.5) in the West African huts $(P<0.001)$. The same trend was observed with Olyset nets, with a median of 7.5 (IQR 3.0-17.5) captured in the West African huts compared to a median of 29.5 (IQR 18.5-37.5) Culex mosquitoes in the Ifakara huts $(P<0.001)$. Deterrence with the low and high spatial repellent metofluthrin dosages was greater in the West African huts (78 and 79\%) than in the Ifakara huts (28 and 49\%), and a dosedependent difference in mosquito entry was measurable in the Ifakara huts (Table 2).

\section{Induced exophily}

Results in Table 3 show that the exophily associated with Olyset nets relative to the control in Ifakara huts (OR: 4.44, 95\% CI: 3.48-5.66, $P<0.001)$ was similar to that of West African huts (OR: 3.91, 95\% CI: 2.84-5.38, $P<$ 0.001). Cx quinquefasciatus mosquito that exit from the Ifakara huts was approximately double that of West
African huts (64 vs 35\%) in the control, odds ratio (OR) 4.18 (95\% CI: $3.18-5.51, P<0.001$ ) (Table 4). This trend was similar across all treatments with consistently greater egress with each treatment from the Ifakara huts than from the West African huts (Table 4).

\section{Blood-feeding rate and personal protection}

The results of blood-feeding rates recorded by both hut designs using the different treatments are presented in Tables 5 and 6 . There were significantly lower bloodfeeding rates of Culex mosquitoes in Ifakara huts with control nets (25\%) than in the West African huts (56\%) (OR: $0.20,95 \%$ CI: $0.15-0.28, P<0.001)$. In both type of huts personal protection was highest with Olyset nets, measured at $96 \%$ in Ifakara huts and $93 \%$ in West African huts although this was statistically different between hut types: (OR: 0.29, 95\% CI: 0.15-0.57, $Z=-3.57$, $P<0.001$ ) (Table 6). The experiment demonstrated a dose-dependent effect on personal protection induced by the metofluthrin coils in the Ifakara huts $(62 \%$

Table 3 Exopily induced by Olyset ${ }^{\oplus}$ Net, metofluthrin coils at $0.00625 \%$ and $0.0097 \%$ and untreated control net in Ifakara huts and West African experimental huts

\begin{tabular}{|c|c|c|c|c|c|c|c|}
\hline & No. caught & Exiting \% $(95 \% \mathrm{Cl})$ & Median IQR exiting & OR & $95 \% \mathrm{Cl}$ & $P$-value & $Z$ \\
\hline \multicolumn{8}{|l|}{ Ifakara huts } \\
\hline Control net & 2067 & $64(62-66)$ & $23(14-33.5)$ & 1 & & & \\
\hline Metofluthrin $0.00625 \%$ & 1489 & $82(80-84)$ & $20(13-28)$ & 2.76 & $2.28-3.35$ & $<0.0001$ & 10.33 \\
\hline Metofluthrin $0.0097 \%$ & 1054 & $85(83-87)$ & $18(10-24)$ & 3.22 & $2.55-4.07$ & $<0.0001$ & 9.84 \\
\hline Olyset $^{\oplus}$ Net & 1658 & $88(86-89)$ & $27.5(17.5-34.5)$ & 4.44 & $3.48-5.66$ & $<0.0001$ & 12.02 \\
\hline \multicolumn{8}{|l|}{ West African huts } \\
\hline Control net & 587 & 35 (31-39) & $2(0.5-3)$ & 1 & & & \\
\hline Metofluthrin $0.00625 \%$ & 122 & $49(40-56)$ & $0(0-2)$ & 2.14 & $1.37-3.34$ & $<0.0001$ & 3.33 \\
\hline Metofluthrin $0.0097 \%$ & 130 & $40(31-48)$ & $0(0-1)$ & 1.52 & $0.98-2.36$ & 0.06 & 1.87 \\
\hline Olyset $^{\oplus}$ Net & 553 & $60(56-64)$ & $4(2-11)$ & 3.91 & $2.84-5.38$ & $<0.0001$ & 8.36 \\
\hline
\end{tabular}


Table 4 Measurements of induced exophily compared between Ifakara and West African experimental huts for control net, Olyset ${ }^{\oplus}$ Net and metofluthrin coils at $0.00625 \%$ and $0.0097 \%$

\begin{tabular}{|c|c|c|c|c|c|c|c|}
\hline Exophily & No. caught & Exiting \% (95\% Cl) & Median IQR exiting & OR & $95 \% \mathrm{Cl}$ & $P$-value & $z$ \\
\hline \multicolumn{8}{|l|}{ Control net } \\
\hline West African hut & 587 & $35(31-39)$ & $2(0.5-3)$ & 1 & & & \\
\hline Ifakara hut & 2067 & $64(62-66)$ & $23(14-33.5)$ & 4.18 & $3.18-5.51$ & $<0.0001$ & 10.18 \\
\hline \multicolumn{8}{|l|}{ Olyset $^{\oplus}$ Net } \\
\hline West African hut & 553 & $60(56-64)$ & $4(2-11)$ & 1 & & & \\
\hline Ifakara hut & 1658 & $88(86-89)$ & $27.5(17.5-34.5)$ & 4.77 & $3.56-6.37$ & $<0.0001$ & 10.53 \\
\hline \multicolumn{8}{|c|}{ Metofluthrin $0.00625 \%$} \\
\hline West African hut & 122 & $49(40-56)$ & $0(0-2)$ & 1 & & & \\
\hline Ifakara hut & 1489 & $82(80-84)$ & 20 (13-28) & 5.41 & $3.54-8.28$ & $<0.0001$ & 7.79 \\
\hline \multicolumn{8}{|c|}{ Metofluthrin $0.0097 \%$} \\
\hline West African hut & 130 & $40(31-48)$ & $0(0-1)$ & 1 & & & \\
\hline Ifakara hut & 1054 & $85(83-87)$ & $18(10-24)$ & 8.88 & 5.74-13.73 & $<0.0001$ & 9.82 \\
\hline
\end{tabular}

protection for low dose and $81 \%$ protection for high dose) (Table 6). However, the dose effect was not seen in the West African huts: 87 vs $89 \%$ personal protection with the lower and higher dose, respectively. The overlapping of the confidence intervals for \% blood-feeding suggests that the West African huts did not discriminate a dose effect.

\section{Induced mortality}

Mosquito mortality induced by all treatments in the Ifakara huts was consistently higher than in the West African huts (Tables 7 and 8). This was particularly pronounced with the high dose metofluthrin coil where mortality was $29 \%$ (95\% CI: 26-32) in the Ifakara huts compared to 3\% (95\% CI: 0.01-6) in the West African huts, odds ratio 13.93 (95\% CI: 4.90-39.63, $P<0.001$ ). Also, mortality in the Ifakara huts with Olyset nets (25\%) (95\% CI: 2-27) was higher than in the West African huts (10\%) (95\% CI: 7-12), odds ratio 3.95 (95\% CI: 2.55-6.12, $P<0.001$ ). Control mortality was also marginally higher in the Ifakara huts (9\%) (95\% CI: 8-10) than the West African huts (6\%) (95\% CI: 4-8); the difference though significant was borderline, odds ratio 1.64 (95\% CI: $1.01-2.66, P=0.04)$ and both values were within acceptable levels as recommended by WHOPES $(<10 \%)$.

\section{Discussion}

The data suggest that the design of each hut type impacts vector behaviour differently. The Ifakara hut with control net was almost four-fold more attractive to $C x$ quinquefasciatus than the West African huts, owing to access of mosquitoes to the hut through the eave gaps (between 50 and $60 \mathrm{~cm}$ wide each) on all four sides compared to entrance via the small window slits $(1 \mathrm{~cm}$ wide each) on three sides of the West African huts.

The analysis of the exophily rates in the Ifakara huts showed that when mosquitoes enter huts naturally for a blood meal source and sleepers in those huts are protected by untreated bednet, a large proportion of hungry

Table 5 Blood-feeding rates of pyrethroid resistant Culex quinquefasciatus in Ifakara and West African experimental huts

\begin{tabular}{|c|c|c|c|c|c|c|c|c|c|}
\hline & No. caught & $\begin{array}{l}\text { Total } \\
\text { blood-fed }\end{array}$ & $\begin{array}{l}\text { Median IQR } \\
\text { blood-fed }\end{array}$ & OR & $95 \% \mathrm{Cl}$ & $P$-value & $Z$ & $\begin{array}{l}\text { Blood-feeding \% } \\
(95 \% \mathrm{Cl})\end{array}$ & $\begin{array}{l}\text { Personal } \\
\text { protection (\%) }\end{array}$ \\
\hline \multicolumn{10}{|l|}{ Ifakara huts } \\
\hline Control net & 2067 & 516 & $8.5(3.5-12)$ & 1 & & & & $25(23-27)$ & - \\
\hline Metofluthrin $0.00625 \%$ & 1489 & 194 & $2(0-5)$ & 0.36 & $0.28-0.46$ & $<0.0001$ & -8.16 & $13(11-15)$ & 62 \\
\hline Metofluthrin $0.0097 \%$ & 1054 & 95 & $1(0-3)$ & 0.33 & $0.25-0.44$ & $<0.0001$ & -7.63 & $9(7-11)$ & 81 \\
\hline Olyset $^{\oplus}$ Net & 1658 & 17 & $0(0-0)$ & 0.03 & $0.02-0.05$ & $<0.0001$ & -13.76 & $1(0.5-1.5)$ & 96 \\
\hline \multicolumn{10}{|l|}{ West African huts } \\
\hline Control net & 587 & 329 & $4.5(1.5-8.5)$ & 1 & & & & $56(52-60)$ & - \\
\hline Metofluthrin $0.00625 \%$ & 122 & 41 & $0(0-1)$ & 0.33 & $0.20-0.54$ & $<0.0001$ & -4.38 & $34(26-42)$ & 87 \\
\hline Metofluthrin $0.0097 \%$ & 130 & 35 & $0(0-1)$ & 0.19 & $0.11-0.31$ & $<0.0001$ & -6.72 & $27(19-35)$ & 89 \\
\hline Olyset $^{\oplus}$ Net & 553 & 22 & $0(0-0)$ & 0.02 & $0.01-0.03$ & $<0.0001$ & -14.24 & $4(2-6)$ & 93 \\
\hline
\end{tabular}


Table 6 Measurements of blood-feeding rates compared between Ifakara huts and West African experimental huts for control net, Olyset $^{\oplus}$ Net and metofluthrin coils at $0.00625 \%$ and $0.0097 \%$

\begin{tabular}{|c|c|c|c|c|c|c|c|c|c|}
\hline & No. caught & $\begin{array}{l}\text { Total } \\
\text { blood-fed }\end{array}$ & Median IQR blood-fed & OR & $95 \% \mathrm{Cl}$ & $P$-value & $Z$ & $\begin{array}{l}\text { Blood-feeding \% } \\
(95 \% \mathrm{Cl})\end{array}$ & $\begin{array}{l}\text { Personal } \\
\text { protection \% }\end{array}$ \\
\hline \multicolumn{10}{|l|}{ Control } \\
\hline West African hut & 587 & 329 & $4.5(1.5-8.5)$ & 1 & & & & $56(52-60)$ & - \\
\hline Ifakara hut & 2067 & 516 & $8.5(3.5-12)$ & 0.20 & $0.15-0.28$ & $<0.0001$ & -10.10 & $25(23-27)$ & - \\
\hline \multicolumn{10}{|l|}{ Olyset ${ }^{\oplus}$ Net } \\
\hline West African hut & 553 & 22 & $0(0-0)$ & 1 & & & & $4(2-6)$ & 93 \\
\hline Ifakara hut & 1658 & 17 & $0(0-0)$ & 0.29 & $0.15-0.57$ & $<0.0001$ & -3.57 & $1(0.5-1.5)$ & 96 \\
\hline \multicolumn{10}{|c|}{ Metofluthrin $0.00625 \%$} \\
\hline West African hut & 122 & 41 & $0(0-1)$ & 1 & & & & $34(26-42)$ & 87 \\
\hline Ifakara hut & 1489 & 194 & $2(0-5)$ & 0.22 & $0.13-0.36$ & $<0.0001$ & -5.96 & $13(11-15)$ & 62 \\
\hline \multicolumn{10}{|c|}{ Metofluthrin $0.0097 \%$} \\
\hline West African hut & 130 & 35 & $0(0-1)$ & 1 & & & & $27(19-35)$ & 89 \\
\hline Ifakara hut & 1054 & 95 & $1(0-3)$ & 0.36 & $0.22-0.60$ & $<0.0001$ & -3.92 & $9(7-11)$ & 81 \\
\hline
\end{tabular}

females (in the order of $64 \%$ in the present trial) exit the huts. The egress from these huts was further increased by the presence of Olyset net or metofluthrin repellent interventions, with induced exophily reaching $88 \%$ for the Olyset net in the Ifakara huts and $60 \%$ in the West African huts. Against An. arabiensis in Tanzania, Olyset net in the Ifakara huts also induced similar exophily rate of 90\% [9] although lower deterrence was observed in the Tanzanian field study than in the study here reported. An explanation could be that a larger number of experimental huts were used in the Tanzanian study over a longer duration that may have reduced the impact of spatial heterogeneity on relative mosquito densities. The lower deterrence in Tanzania could also be due to species differences; $C x$. quinquefasciatus in the current Beninese trial versus An. arabiensis in Tanzania. Studies have shown that $A n$. arabiensis is likely to exit huts when a blood meal source is protected by a bednet [9]. The present study shows a similar trend for $C x$. quinquefasciatus with only blood-fed mosquitoes remaining inside the Ifakara huts. The consequence is that the proportions of mosquitoes remaining inside and killed in the Ifakara huts were small (25\% of resistant Culex with Olyset net), a rate higher than that killed by Olyset net in Tanzania (4\% Culex mortality) in same hut type [9]. In the West African hut, Olyset net killed only $10 \%$ of Culex mosquitoes, a rate similar to previous trials conducted in the same study area [8]. The proportion of Culex mosquitoes dying in the presence of Olyset net was higher in the Ifakara hut than in the West African hut. This finding is at odds with a similar study comparing Olyset net in a side by side comparison of the Ifakara and the West African huts in Tanzania where mortality did not differ [18]. However, control mortality in this study was unacceptably high (41\%) so the insecticide induced mortality could not be detected, $v s<10 \%$ in our current study, excluding any comparison between the two studies. Moreover, the performance of the three

Table 7 Mortality induced by Olyset ${ }^{\oplus}$ Net, metofluthrin coils at $0.00625 \%$ and $0.0097 \%$ and untreated control net in Ifakara and West African experimental huts

\begin{tabular}{|c|c|c|c|c|c|c|c|c|}
\hline & No. caught & Total dead & Median IQR dead & OR & $95 \% \mathrm{Cl}$ & $P$-value & $Z$ & Mortality \% (95\% Cl) \\
\hline \multicolumn{9}{|l|}{ Ifakara huts } \\
\hline Control net & 2067 & 195 & $2(0-5.5)$ & 1 & & & & $9(8-10)$ \\
\hline Metofluthrin 0.00625\% & 1489 & 204 & $3(0-6)$ & 1.71 & $1.33-2.20$ & $<0.0001$ & 4.14 & $14(12-16)$ \\
\hline Metofluthrin $0.0097 \%$ & 1054 & 307 & $5(1-10)$ & 4.46 & $3.44-41.83$ & $<0.0001$ & 11.29 & $29(26-32)$ \\
\hline Olyset Net ${ }^{\oplus}$ & 1658 & 423 & $7(5-11)$ & 4.12 & $3.13-5.78$ & $<0.0001$ & 10.09 & $25(2-27)$ \\
\hline \multicolumn{9}{|l|}{ West African huts } \\
\hline Control net & 587 & 35 & $0(0-0.5)$ & 1 & & & & $6(4-8)$ \\
\hline Metofluthrin $0.00625 \%$ & 122 & 12 & $0(0-0)$ & 1.49 & $0.71-3.12$ & 0.29 & 1.05 & $10(5-15)$ \\
\hline Metofluthrin $0.0097 \%$ & 130 & 4 & $0(0-0)$ & 0.52 & $0.18-1.53$ & 0.23 & -1.19 & $3(0.1-6)$ \\
\hline Olyset Net ${ }^{\oplus}$ & 553 & 53 & $0(0-1)$ & 1.69 & $1.01-2.84$ & 0.05 & 1.99 & $10(7-12)$ \\
\hline
\end{tabular}


Table 8 Measurements of mortality compared between Ifakara and West African experimental huts for control net, Olyset ${ }^{\circledR}$ Net and metofluthrin coils at $0.00625 \%$ and $0.0097 \%$

\begin{tabular}{|c|c|c|c|c|c|c|c|c|}
\hline Mortality & No. caught & Total dead & Median IQR dead & OR & $95 \% \mathrm{Cl}$ & $P$-value & $Z$ & Mortality \% (95\%Cl) \\
\hline \multicolumn{9}{|l|}{ Control } \\
\hline West African hut & 587 & 35 & $0(0-0.5)$ & 1 & & & & $6(4-8)$ \\
\hline Ifakara hut & 2067 & 195 & $2(0-5.5)$ & 1.64 & $1.01-2.66$ & 0.04 & 2.01 & $9(8-10)$ \\
\hline \multicolumn{9}{|l|}{ Olyset $^{\oplus}$ Net } \\
\hline West African hut & 553 & 53 & $0(0-1)$ & 1 & & & & $10(7-12)$ \\
\hline Ifakara hut & 1658 & 423 & $7(5-11)$ & 3.95 & $2.55-6.12$ & $<0.0001$ & 6.14 & $25(2-27)$ \\
\hline \multicolumn{9}{|c|}{ Metofluthrin $0.00625 \%$} \\
\hline West African hut & 122 & 12 & $0(0-0)$ & 1 & & & & $10(5-15)$ \\
\hline Ifakara hut & 1489 & 204 & $3(0-6)$ & 1.85 & $0.92-3.72$ & 0.08 & 1.71 & $14(12-16)$ \\
\hline \multicolumn{9}{|c|}{ Metofluthrin $0.0097 \%$} \\
\hline West African hut & 130 & 4 & $0(0-0)$ & 1 & & & & $3(0.1-6)$ \\
\hline Ifakara hut & 1054 & 307 & $5(1-10)$ & 13.93 & $4.90-39.63$ & $<0.0001$ & 4.94 & $29(26-32)$ \\
\hline
\end{tabular}

experimental huts tested in the Tanzanian study was assessed on a pooled set of mosquitoes [An. gambiae (s.l.), An. funestus, Mansonia and Culex mosquitoes] rather than presenting the data by species, again making the comparison of mortality measurements between the two studies impossible.

In the current study, the significant difference in mortality observed when Olyset ${ }^{\circ}$ Net was tested in the Ifakara hut (25\%) vs in the West African hut (10\%) is a result of the difference in hut structure. First, the roof of the Ifakara hut is surrounded by eaves and has four window exit traps, which increased the exit rate of mosquitoes after an unsuccessful attempt to blood-feed. This is supported by our data which showed greater exophily rate with this hut type. The mosquitoes are subsequently trapped in the exit space where they likely died from starvation. This might account for the higher mortality with the Olyset net in the Ifakara hut. In future studies, it may be useful to provide mosquitoes with a sugar solution to prevent starvation. This difference in hut structure where mosquitoes are retained in the West African huts until morning may have impacted blood-feeding estimate with mosquitoes more likely to make multiple attempts to blood-feeding rather than exiting once they fail to feed. This would seem unrealistic, particularly within those rural households that have windows and eaves that allow mosquitoes to freely enter and exit, and consequently may not reflect the natural expression of the mosquitoes behaviour, especially where users are protected with nets.

The usual egress rates of Culex mosquitoes as estimated in the West African hut in the absence of interventions from several trials averaged 30\% [19-22] compared to $64 \%$ in the current study and over $90 \%$ in the previous study [9]. It is understandable that a good rationale behind the West African design is fixing the hut tight that is also based on the style of houses used in Burkina Faso to allow a more accurate estimation of the denominator in measuring the different entomological parameters, e.g. the total number of mosquitoes that initially entered that hut. However, the Ifakara huts have eave gaps and prevent egress of mosquitoes via the eaves using baffles [2], so the mosquitoes are retained within the huts or captured in eave and window exit traps.

The West African huts could be easily amended to accommodate natural exit route of mosquitoes that best reflects reality. For example, one could overcome mosquito restriction within huts by concrete-sealing the verandah compartment and funnelling slits on the top wall of that verandah compartment for the mosquitoes to exit the huts freely but limit their return. A small door designed onto the meshed area projected on the back of that verandah will allow morning collection and a good estimate of mosquitoes that would normally have escaped the huts.

While deterrence induced by the repellent interventions (coils) was apparent in both hut styles the effect was more pronounced in the West African hut design (78-79\%) compared to the effect observed in Ifakara huts $(28-49 \%)$. Since mosquitoes are forced to enter the huts through narrow slits, it is likely that the concentration of volatile pyrethroid encountered will be far higher than in the larger and more open Ifakara huts. The airflow of an experimental hut is an important consideration when considering vapour acting interventions, and the Ifakara huts were shown to have similar indoor air movements as local houses [12]. Also, when considering the impact of indoor residual spray (IRS), it is important to consider the temperature and airflow inside of experimental huts as this will lead to volatilization of insecticide particles [23]. Unfortunately, comparison of temperature and wind movement between huts used in this study was not made. 
This study showed differences in huts feature between West and Ifakara huts; however, both types are equivalently suitable to evaluate products of any kind, i.e. whether insecticidal or repellent. WHO recommends that a novel product should be assessed alongside a positive control of the same family products against the same vector species at a given site and type of hut; it should always be possible to evaluate the relative efficacy of a product in a given type of hut despite the structural difference in feature.

\section{Conclusions}

The data show clear differences between parameters measured by each hut with each parameter being consistently differently measured for the control, Olyset ${ }^{\circ}$ Net and spatial repellents. Of particular importance is the fact that the Ifakara huts caught a significantly higher number of $C x$ quinquefasciatus mosquitoes and allowed free exit of mosquitoes from huts when they do not obtain a blood meal whereas fewer mosquitoes can enter or leave the West African huts. This is an important consideration when evaluating interventions that require mosquito behaviour to be as normal as possible.

\section{Abbreviations}

AIC: Akaike's information criterion; Cl: Confidence interval;

GLMMs: Generalized linear mixed models; IQR: Interquartile range; IRS: Indoor residual spray; kdr: Knockdown resistance; LLIN: Long-lasting insecticidal nets; OR: Odds ratio; WHO: World Health Organisation; WHOPES: World Health Organisation Pesticide Evaluation Scheme

\section{Acknowledgements}

The study was funded by Bill and Melinda Gates Foundation Grant 51431.This funding body played no role in the design of the study and collection, analysis, and interpretation of data and in writing the manuscript. The authors would like to thank Professor Martin Akogbeto for assistance in setting up the study and for Dennis J Massue for assisting with manuscript editing. Our gratitude also goes to the volunteer residents of Donoukin for taking part in the trial.

\section{Funding}

This research was supported by the Bill and Melinda Gates Foundation.

\section{Availability of data and materials}

The data sets supporting the conclusions of this article are included in this article. The raw data can be obtained from the corresponding author on reasonable request.

\section{Authors' contributions}

WAO and AF conducted the field trials. WAO drafted the manuscript. KBS helped with the data analysis. SJM conceived and designed the experiment, analysed the data and contributed to writing the manuscript. RN aided in the study design, supervised the project and co-wrote the paper. All authors read and approved the final manuscript.

\section{Ethics approval and consent to participate}

Ethical approval for the study was granted by the Ethics Committee within the Ministry of Health in Benin. Written informed consent to participate in the study was obtained from all volunteers before the commencement of the trial.

\section{Consent for publication}

Not applicable.

\section{Competing interests}

The authors declare that they have no competing interests.

\section{Publisher's Note}

Springer Nature remains neutral with regard to jurisdictional claims in published maps and institutional affiliations.

\section{Author details}

${ }^{1}$ Centre de Recherche Entomologique de Cotonou, Cotonou, Benin. 'Environmental Health and Ecological Sciences Thematic Group, Ifakara Health Institute, Bagamoyo Research and Training Centre, Bagamoyo, Tanzania. ${ }^{3}$ Swiss Tropical and Public Health Institute, Socinstr., 574051 Basel, Switzerland. ${ }^{4}$ University of Basel, Petersplatz 1, 4003 Basel, Switzerland.

${ }^{5}$ London School of Hygiene and Tropical Medicine, Keppel Street, WC1E 7HT London, UK.

Received: 13 May 2017 Accepted: 7 September 2017

Published online: 19 September 2017

References

1. Smith A, Hudson JE, Obudho O. Preliminary louvre-trap hut studies on the egress of Anopheles gambiae Giles, Mansonia uniformis (Theo.) and Culex pipiens fatigans Wied. from untreated huts. Bull Entomol Res. 1972;61:415-29.

2. Darriet F, N'Guessan R, Hougard JM, Traoré-Lamizana M, Carnevale P. An experimental tool essential for the evaluation of insecticides: the testing huts. Bull Soc Pathol Exot. 2002;95:299-303.

3. Okumu FO, Moore J, Mbeyela E, Sherlock M, Sangusangu R, Ligamba G, et al. A modified experimental hut design for studying responses of disease-transmitting mosquitoes to indoor interventions: the Ifakara experimental huts. PLoS One. 2012;7:e30967.

4. Achee NL, Grieco JP, Andre RG, Rejmankova E, Roberts DR. A mark-releaserecapture study using a novel portable hut design to define the flight behavior of Anopheles darlingi in Belize, central America. J Am Mosq Control Assoc. 2005;21:366-79.

5. Smith A, Hudson JE. A modification to an experimental hut to reduce mosquito eaves-egress. WHO/mal/72.775. In: WHO/Mal/72.775, editor. A modification to an experimental hut to reduce mosquito eaves-egress. Geneva: World Health Organisation; 1972. p. 6.

6. Smith A. A review of the origin and development of experimental hut techniques used in the study of insecticides in East Africa. East Afr Med J. 1964:41:361-74.

7. Smith A. A verandah-trap hut for studying the house-frequenting habits of mosquitoes and for assessing insecticides. II. The effect of dichlorvos (DDVP) on egress and mortality of Anopheles gambiae Giles and Mansonia uniformes (Theo.) entering naturally. Bull Entomol Res. 1965;56:275-82.

8. Ngufor CA, N'Guessan R, Fagbohoun J, Odjo A, Malone D, Agobeto M, et al. Olyset duo ${ }^{\oplus}$ (a pyriproxyfen and permethrin mixture net). An experimental hut trial against pyrethroid resistant Anopheles gambiae and Culex quinquefasciatus in southern Benin. PLoS One. 2014:9:93603.

9. Okumu FO, Mbeyela E, Lingamba G, Moore J, Ntamatungiro AJ, Kavishe DR, et al. Comparative field evaluation of combinations of long-lasting insecticide treated nets and indoor residual spraying, relative to either method alone, for malaria prevention in an area where the main vector is Anopheles arabiensis. Parasit Vectors. 2013:6:46.

10. N'Guessan R, Corbel V, Akogbeto M, Rowland M. Reduced efficacy of insecticide-treated nets and indoor residual spraying for malaria control in pyrethroid resistance area, Benin. Emerg Infect Dis. 2007;13:199-206.

11. Deparis $X$, Frere $B$, Lamizana $M, N^{\prime}$ 'Guessan $R$, Leroux $F$, Lefevre $P$, et al. Efficacy of permethrin-treated uniforms in combination with DEET topical repellent for protection of French military troops in cote d'Ivoire. J Med Entomol. 2004;41:914-21.

12. Ogoma SB, Lorenz LM, Ngonyani $H$, Sangusangu R, Kitumbukile $M$, Kilalangongono $\mathrm{M}$, et al. An experimental hut study to quantify the effect of DDT and airborne pyrethroids on entomological parameters of malaria transmission. Malar J. 2014;13:131.

13. Massue DJ, Kisinza WN, Malongo BB, Mgaya CS, Bradley J, Moore JD, et al. Comparative performance of three experimental hut designs for measuring malaria vector responses to insecticides in Tanzania. Malar J. 2016;15:165.

14. Djouaka RF, Bakare AA, Coulibaly ON, Akogbeto MC, Ranson H, Hemingway J, et al. Expression of the cytochrome P450s, CYP6P3 and CYP6M2 are significantly elevated in multiple pyrethroid resistant populations of 
Anopheles gambiae s.s. from southern Benin and Nigeria. BMC Genomics. 2008;9:538.

15. Corbel V, N'Guessan R, Brengues C, Chandre F, Djogbenou L, Martin T, et al. Multiple insecticide resistance mechanisms in Anopheles gambiae and Culex quinquefasciatus from Benin, West Africa. Acta Trop. 2007;101:207-16.

16. WHO. Guidelines for laboratory and field-testing of long-lasting insecticidal nets. Geneva: World Health Organization; 2013.

17. R Development Core Team R. A language and environment for statistical computing. Vienna: R Foundation for Statistical, Computing; 2011.

18. Bates D, Maechler M, Bolker B. Ime4: Linear mixed-effects models using $\mathrm{S} 4$ Classes. R package version 0999375-39. 2011. http://CRAN.R-project.org/ package $=$ Ime4. Accessed 2016

19. Asidi AN, N'Guessan R, Koffi AA, Curtis CF, Hougard J-M. Fabrice Chandre, et al. experimental hut evaluation of bednets treated with an organophosphate (chlorpyrifos-methyl) or a pyrethroid (lambdacyhalothrin) alone and in combination against insecticide-resistant Anopheles gambiae and Culex quinquefasciatus mosquitoes. Malar J. 2005:4:25.

20. N'Guessan R, Boko P, Odjo A, Knols B, Akogbeto M, Mark R. Control of pyrethroid-resistant Anopheles gambiae and Culex quinquefasciatus mosquitoes with chlorfenapyr in Benin. Trop Med Int Health. 2009;14:389-95.

21. N'Guessan R, Asidi A, Boko P, Odjo A, Akogbeto M, Pigeon O, et al. An experimental hut evaluation of $\operatorname{PermaNet}\left({ }^{\circ}\right)$ 3.0, A deltamethrinpiperonylbutoxide combination net, against pyrethroid-resistant Anopheles gambiae and Culex quinquefasciatus mosquitoes in southern Benin. Trans R Soc Trop Med Hyg. 2010;104:758-65.

22. Akogbéto MC, Padonou GG, Gbénou D, Irish S, Yadouleton A. Bendiocarb, a potential alternative against pyrethroid resistant Anopheles gambiae in Benin. West Africa Malar J. 2010;9:204.

23. Fleck EE. Rate of evaporation of DDT. J Econ Entomol. 1944;37:853.

\section{Submit your next manuscript to BioMed Central and we will help you at every step:}

- We accept pre-submission inquiries

- Our selector tool helps you to find the most relevant journal

- We provide round the clock customer support

- Convenient online submission

- Thorough peer review

- Inclusion in PubMed and all major indexing services

- Maximum visibility for your research

Submit your manuscript at www.biomedcentral.com/submit 